Brazilian Journal of Forensic Sciences, Medical Law and Bioethics

Journal homepage: www.ipebj.com.br/forensicjournal

\title{
Mentira na Perícia Psicológica: Avaliação da Atitude Malinger numa Amostra Forense
}

\section{Liying in Psychological Assessments: Evaluation of the Malinger Attitude in a Forensic Sample}

\author{
Francisco Valente Gonçalves ${ }^{1, \star}$, Rui Mateus Joaquim², Sara Ferreira ${ }^{3}$, \\ Miguel Ângelo ${ }^{4}$, Leonardo Ferreira Faria ${ }^{5}$ \\ ${ }^{1}$ EY Forensic \& Integrity Services, Lisboa, Portugal \\ ${ }^{2}$ Instituto Pauista de Estudos Bioéticos e Jurídicos (IPEBJ), Ribeirão Preto, SP, Brasil \\ ${ }^{3}$ Faculdade de Ciências da Universidade de Lisboa, Lisboa, Portugal \\ ${ }^{4}$ Instituto Superior de Psicologia Aplicada, Instituto Universitário, Lisboa, Portugal \\ ${ }^{5}$ Polícia Técnico-Científica do Estado de Goiás, Goiânia, Goiás, Brasil
}

Received 16 July 2020

Resumo. Aquando necessário avaliar-se a atitude falsa, como por exemplo a mentira, numa perícia forense, os profissionais que realizam este tipo de trabalho têm limitados os recursos a que podem recorrer. Uma das razões prende-se com o diminuto investimento na investigação na área da avaliação da mentira. Ainda sobre este ponto, avaliar a mentira é uma tarefa extremamente complexa, sendo vários os olhares que um poderá ter para realizar tal ação. Uma possível forma de observar atitudes falsas é a análise da presença de atitudes malinger. No entanto as ferramentas de avaliação a nível psicométrico para esta área são também escassas e pouco elegantes a nível científico. Finalmente, e mais importante, observa-se uma falta de investigação onde as amostras são totalmente forenses. O presente trabalho apresenta uma descrição de uma amostra forense $(n=470)$ relativamente ao tipo de atitude malinger presente e às características sociodemográficas da mesma. Observam-se alguns pontos de interesse em alguns tipos de características sociais tais como o tipo de habilitação académica ou o tipo de profissão. O tipo de processo jurídico a que os sujeitos em avaliação estão afetos parece ser importante de considerar e finalmente observa-se uma correlação de cerca de 13\% em que a inteligência dos sujeitos explica a sua atitude malinger. Mais estudos são necessários. Os dados apresentados neste trabalho são do interesse dos profissionais da especialidade, mas acima de tudo, são 
acreditados como um passo para se ter uma linha de comparação para investimento científico no futuro na presente área de avaliação forense.

Palavras-chave: Mentira; Malingering; Perícia Psicológica.

Abstract. When it is necessary to evaluate the false attitude, such as lying, in a forensic examination, the professionals who carry out this type of work have limited resources to which they can resort. One of the reasons is the small investment in research in the area of lie evaluation. Still on this point, evaluating the lie is an extremely complex task, with several views that one may have to carry out such action. One possible way to observe false attitudes is to analyze the presence of malinger attitudes. However, psychometric assessment tools for this area are also scarce and not very scientifically elegant. Finally, and most importantly, there is a lack of investigation where the samples are entirely forensic. The present work presents a description of a forensic sample $(n=470)$ regarding the type of malinger attitude present and its sociodemographic characteristics. There are some points of interest in some types of social characteristics such as the type of academic qualification or the type of profession. The type of legal process to which the subjects being assessed seem to be important to consider and finally there is a correlation of about $13 \%$ in which the subjects' intelligence explains their malinger attitude. Further studies are needed. The data presented in this work are of interest to professionals in the specialty, but above all, they are believed as a step towards having a line of comparison for scientific investment in the future in this area of forensic evaluation.

Keywords: Lying; Malingering; Psychological Assessment.

\section{Introdução}

A recolha de informação em momento pericial obedece a um protocolo de entrevista [por norma semiestruturada] onde se encontram pontos relativos à observação clínica do comportamento, registos biográficos do sujeito, técnicas estandardizadas [e.g., testes e/ou questionários] ${ }^{1,2}$ e potenciais fontes colaterais ${ }^{3,4,5}$. Ao contrário do contexto clínico, é esperado que o examinando tenda a distorcer informações sobre si, com o objectivo de alcançar eventuais benefícios. Sendo esta distorção realizada de forma consciente, está-se perante o reconhecido fenómeno designado na literatura por malingering ${ }^{6,7}$. Segundo o DSM- $5^{8}$ (pp. 726-727), o conceito de malingering é definido como "the intentional production of false or grossly exaggerated physical or psychological symptoms, motivated by external incentives such as avoiding military duty, avoiding work, obtaining financial compensation, 
evading criminal prosecution, or obtaining drugs". Descrita como uma forma de adaptação ao meio em que o sujeito avalia o custo-benefício das suas opções em contextos adversos ${ }^{9}$, a atitude malinger estima-se que tenha uma prevalência na prática clínica civil de $1 \%{ }^{10}$. Já em contexto militar este valor sobe para $5 \%{ }^{1}$. Finalmente num contexto forense, a prevalência de atitudes malinger aumenta significativamente e pode variar entre $10 \%$ e $20 \%{ }^{1}$. Existem três tipos de atitude malinger segundo Resnick, West e Payne ${ }^{11}$ e Taylor, Fruch e Asmundson ${ }^{12}$ : 1. puro - quando o indivíduo finge uma perturbação que não existe; 2. parcial - quando o indivíduo exagera os sintomas já existentes; e 3. falsa imputação - quando o indivíduo atribui aos seus sintomas, de forma intencional, uma causa que não se relaciona com a origem dos mesmos. Relativamente à forma do conteúdo presente na atitude malinger, Singh e colaboradores ${ }^{1}$ descreveram duas formas possíveis de observar: 1. pura ou parcial - quando ocorre produção [fictícia] de sintomas ou quando existe um exagero na sintomatologia já existente; e 2. positiva ou negativa quando o sujeito finge os sintomas de uma perturbação ou esconde os sintomas de uma perturbação já existente. No presente trabalho, o foco de investigação prendeuse com a observação de atitudes malinger positivas e negativas durante as perícias psicológicas em âmbito forense.

De forma a aferir a presença de uma atitude malinger e garantir a validade dos depoimentos de um sujeito que vai a julgamento, Kitchen ${ }^{13}$ sugere três etapas: 1. ouvir o relato do sujeito; 2. ouvir o relato de terceiros acerca do sujeito; e 3. recorrer ao testemunho de profissionais de saúde mental. Quanto ao testemunho de peritos de psicologia, e realçando a avaliação psicológica, esta é tanto mais fidedigna quanto a multidisciplinariedade, validade e pertinência dos instrumentos que o perito utiliza ${ }^{12}$. Vários autores ${ }^{3,4,7,10,14-17}$ têm investigado e encontrado resultados que suportam a eficácia do uso de testes psicométricos onde as suas escalas de validade complementam a análise de atitudes malinger como é o caso do Inventário Multifásico de Personalidade Minnesota $(\mathrm{MMPI})^{18} \mathrm{e}$ das suas versões reduzidas como o inventário Mini-mult ${ }^{19}$ onde 0 índice $f-k$ apresenta a validade da prova e a possibilidade de sujeitos falsearem as suas atitudes de forma simulada [malinger positivo] ou dissimulada [malinger negativo].

A deteção de comportamentos falsos é uma tarefa extremamente complexa e muitas das vezes suscita dúvidas e enviesamentos aquando o processo de avaliação, sendo que potenciais erros tendem a aumentar se os instrumentos 
aplicados são inadequados e/ou pouco precisos ${ }^{20}$. Ainda, outro fator que poderá observar-se a sua contribuição para a ocorrência de erros na avaliação de comportamentos falsos são os julgamentos morais [i.e. enviesamentos cognitivos] para com o indivíduo avaliado, assim como o conhecimento, por vezes, escasso das várias formas de expressão destes comportamentos de acordo com o contexto e as características culturais do próprio sujeito ${ }^{21}$.

Sendo um dos maiores obstáculos na atualização e progresso da investigação na área do reconhecimento e análise de comportamentos e atitudes falsas, a falta de investimento em investigação com populações forenses, bem como a necessidade de validar provas específicas para este tipo de populações ou investimento em novas técnicas ${ }^{3,21}$, o presente trabalho propõe-se a realizar uma análise e descrição das atitudes malinger numa amostra totalmente forense. Acreditamos que os resultados aqui apresentados irão permitir a profissionais da especialidade enriquecer o conhecimento nesta temática, iniciando-se também a construção de uma linha de comparação que se venha a utilizar como ponto de referência em estudos futuros. É essencial aprofundar o conhecimento acerca das populações forenses que possam potencialmente produzir uma atitude malinger, não só para distinguir as mesmas, mas igualmente para compreender as suas características e motivações a fim de prevenir erros críticos que promovam avaliações periciais imprecisas e inadequadas, finalizando-se as mesmas em decisões judiciais com potenciais lacunas na sua fundamentação para com sujeitos [quer vítimas ou arguidos/as] que integram os processos jurídicos.

\section{Questões de investigação}

As perguntas elaboradas têm o seu foco na atitude malinger de forma geral em primeiro lugar, tendo sido posteriormente realizada uma distinção entre a atitude malinger positiva e a atitude malinger negativa.

Q1. É possível explorar um perfil de pessoas sujeitas a uma perícia forense relativamente à sua atitude malinger?

a. Qual a ocorrência de atitudes malinger consoante o tipo de características sociodemográficas dos sujeitos avaliados?

b. Quais os traços de personalidade com maior presença nos sujeitos que apresentam uma atitude malinger? 
Q2. Serão os tipos de processo e de caso jurídico fonte motivadora para um sujeito apresentar uma atitude malinger durante a perícia forense?

a. Sujeitos afectos a processos onde são requisitadas perícias à personalidade têm uma atitude malinger positiva com maior expressão quando comparados com sujeitos afectos aos restantes casos e tipos de perícia.

b. A atitude malinger negativa é distinta em perícias associadas à avaliação de competências parentais (perícia OTM e 178) quando comparada com outros tipos de processo e tipos de perícia.

Q3. Será que um sujeito com maior QI terá uma maior predisposição para apresentar uma atitude malinger declarada e observável?

a. É possível observar-se uma relação entre o QI dos sujeitos e a escala de validade $f_{-k}$

\section{Metodologia}

\subsection{Amostra}

Foram analisadas as provas psicométricas de 470 perícias psicológicas realizadas por ordem judicial pelo serviço de psicologia do Instituto Nacional de Medicina Legal e Ciências Forenses - Delegação Sul e pelas equipas da Direcção Geral de Reinserção Social - EP Lisboa e Lisboa PENAL2. Todos os processos adjacentes às perícias analisadas tinham o seu curso findado e decisões judiciárias tomadas aquando a análise dos relatórios periciais. Todas as informações relativas aos sujeitos a quem fora realizada a atividade pericial foram anonimizadas aquando a análise dos relatórios periciais.

A idade mínima e máxima de sujeitos avaliados era 17 e 92 anos respectivamente $(\chi=41, S D=13.03)$. A maioria dos sujeitos tinha nacionalidade Portuguesa (83\%), sendo os restantes de nacionalidades várias que não são descriminadas neste trabalho por insignificância estatística. A amostra constituiu-se por 224 homens e 246 mulheres. O estado civil dos sujeitos dividiu-se entre: solteiro (40\%), casado/união de facto (29\%), divorciado (27\%) e viúvo (4\%). As habilitações literárias dos sujeitos avaliados eram distintas à data das avaliações, existindo sujeitos sem qualquer habilitação literária (10\%), outros com habilitações ao nível básico (52\%), ensino secundário (17\%) e ensino superior (21\%). Relativamente ao tipo de trabalho, os sujeitos avaliados foram organizados segundo a Classificação 
Portuguesa das Profissões, resultando uma lista de 10 categorias profissionais tendo sido adicionadas três outras [estudantes, desempregados e reformados] pelos autores do presente trabalho.

Relativamente ao tipo de perícia em que os sujeitos avaliados estavam afetos, foram consideradas cinco categorias: perícia psicológica complementar à perícia psiquiátrica [art. 159 CPP] (36\%), perícia psicológica ao abrigo da Lei no 147/99 [Lei de Proteção de Crianças e Jovens em Perigo] (11\%), perícia psicológica para avaliação do dano em sede de Direito Penal e Civil (6\%), perícia psicológica ao abrigo do artigo 178을 do Decreto-Lei no314/78 (OTM) e da Lei oㅜ 61/2008 (Regime Jurídico do Divórcio) (36\%) e perícia sobre a personalidade [art. 160 CPP] (11\%).

\subsection{Instrumentos}

Das perícias analisadas, foram analisados em específico dois instrumentos aplicados durante o momento de avaliação pericial: Versão Reduzida do Minnesota Multifasic Personality Inventory [MMPI] - Mini-Mult (19) e Matrizes Progressivas de Raven $^{22}$. O primeiro instrumento possibilita a avaliação de traços de personalidade através de oito escalas clínicas bem como a observação de eventuais atitudes comportamentais falsas através das escalas de validade $f$ e $k$. Os manuais desta prova referem que a atitude malinger positiva [produção/simulação falsa de sintomatologia psicopatológica] tem o seu ponto de corte quando $f=k>11$ e a atitude negativa [ocultação/dissimulação de sintomas psicopatológicos] quando $t=k$ $\leq \_15^{23}$. A segunda prova utilizada tem como objetivo inferir sobre a inteligência de indivíduos através da sua capacidade de raciocínio fluído ${ }^{22}$. No uso das Matrizes de Raven, o ponto de corte foi o estipulado pelo manual da prova [Inteligência Normal Corrente - Q.I. > 89].

\subsection{Procedimento}

Posteriormente à avaliação e aceitação ética de objetivos e procedimentos a utilizar durante a recolha de dados e autorização da mesma pelas direcções do Instituto Nacional de Medicina Legal e Ciências Forenses - Delegação Sul e da Direção Geral de Reinserção Social, foi delineado um plano de trabalho calendarizado para realizar as visitas aos arquivos e recolher os dados. Apenas um critério de exclusão foi elaborado no início da recolha pelas direções das instituições onde decorreu a análise de relatórios: seria apenas possível analisar relatórios de perícias onde as 
decisões judiciais teriam sido já aplicadas. Foram selecionadas 475 perícias disponíveis após este critério. Adicionou-se um segundo critério de exclusão após verificar que na maioria das perícias disponíveis para consulta, o teste Mini-Mult fora sempre utilizado. Desta forma, foram excluídas cinco perícias onde não fora utilizado o teste referido, perfazendo o total de 470 perícias analisadas. Da amostra total, a prova Matrizes Progressivas de Raven fora utilizada em $74 \%$ das perícias em análise ( $n=346)$.

As visitas foram realizadas com supervisão institucional. Os arquivos foram deixados na mesma forma que foram encontrados relativamente à sua organização. Durante as visitas aos arquivos os dados de interesse foram localizados e transferidos para uma base de dados onde detalhes pessoais dos indivíduos avaliados não foram transcritos. Os dados de interesse foram codificados e encriptados para posterior análise apenas por um dos autores do presente estudo a fim de manter a segurança dos mesmos. Finalmente, os dados foram analisados com o auxílio do software IBM SPSS Statistics ${ }^{24}$.

\section{Resultados}

\subsection{Q1 (A)}

Existem $27 \%$ de sujeitos que apresentam uma atitude malinger [positiva ou negativa] e $73 \%$ de sujeitos em que não se observaram nenhum dos tipos de atitude malinger durante a aplicação das provas psicométricas realizadas nas perícias, como mostra a Figura 1. Relativamente aos resultados da primeira questão, apresentamos as características sociodemográficas género, habilitações literárias, profissão, estado civil, idade, e nacionalidade respetivamente nas Figuras 2-7.

Os resultados de atitudes malinger positivas e negativas são semelhantes entre o género feminino e masculino. Observam-se ligeiras diferenças onde 0 género masculino tem uma elevação de $3 \%$ na atitude malinger negativa, e de $2 \%$ na atitude malinger positiva relativamente ao género feminino.

Relativamente às habilitações académicas, população onde a atitude malinger negativa teve uma preponderância maior (56\%) deteve-se nos sujeitos com graus académicos do ensino superior. Observam-se ainda 3,7\% com uma atitude malinger positiva cujas habilitações académicas seriam referentes ao ensino básico. 


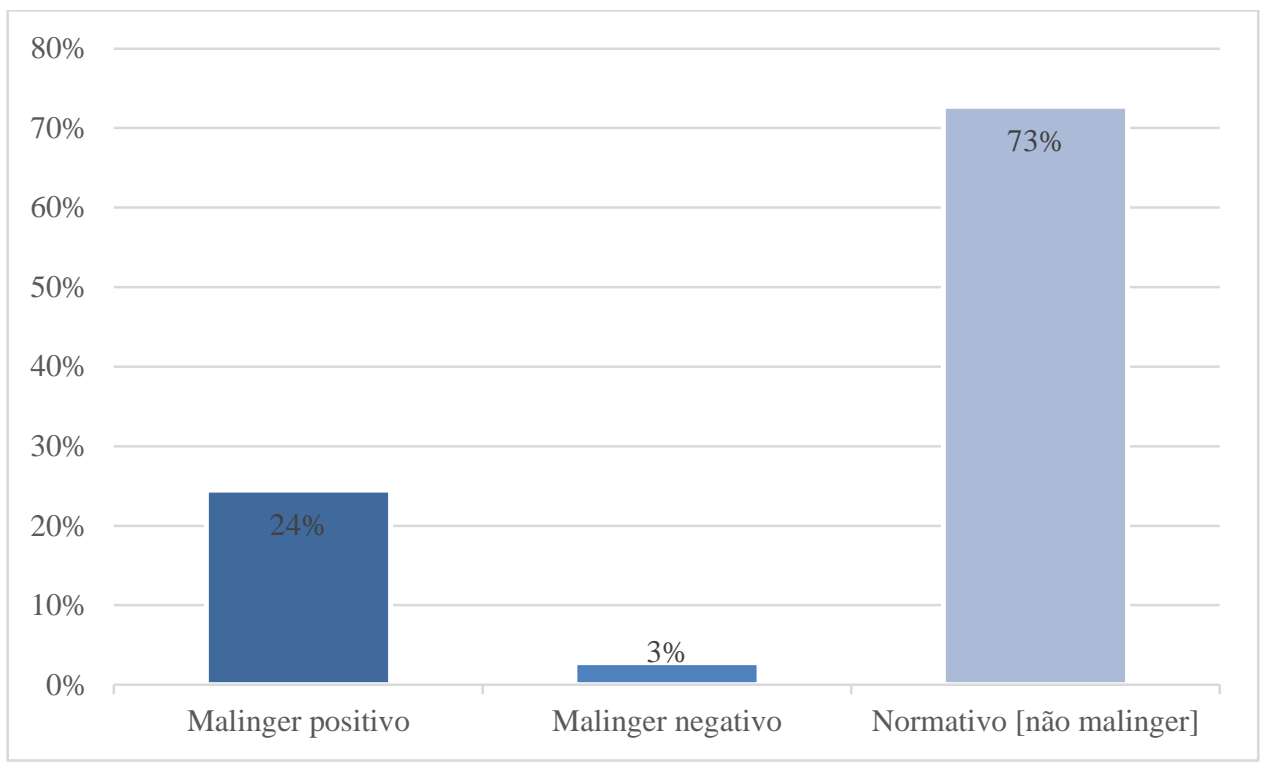

Figura 1. Atitude malinger $(\mathrm{n}=470)$.

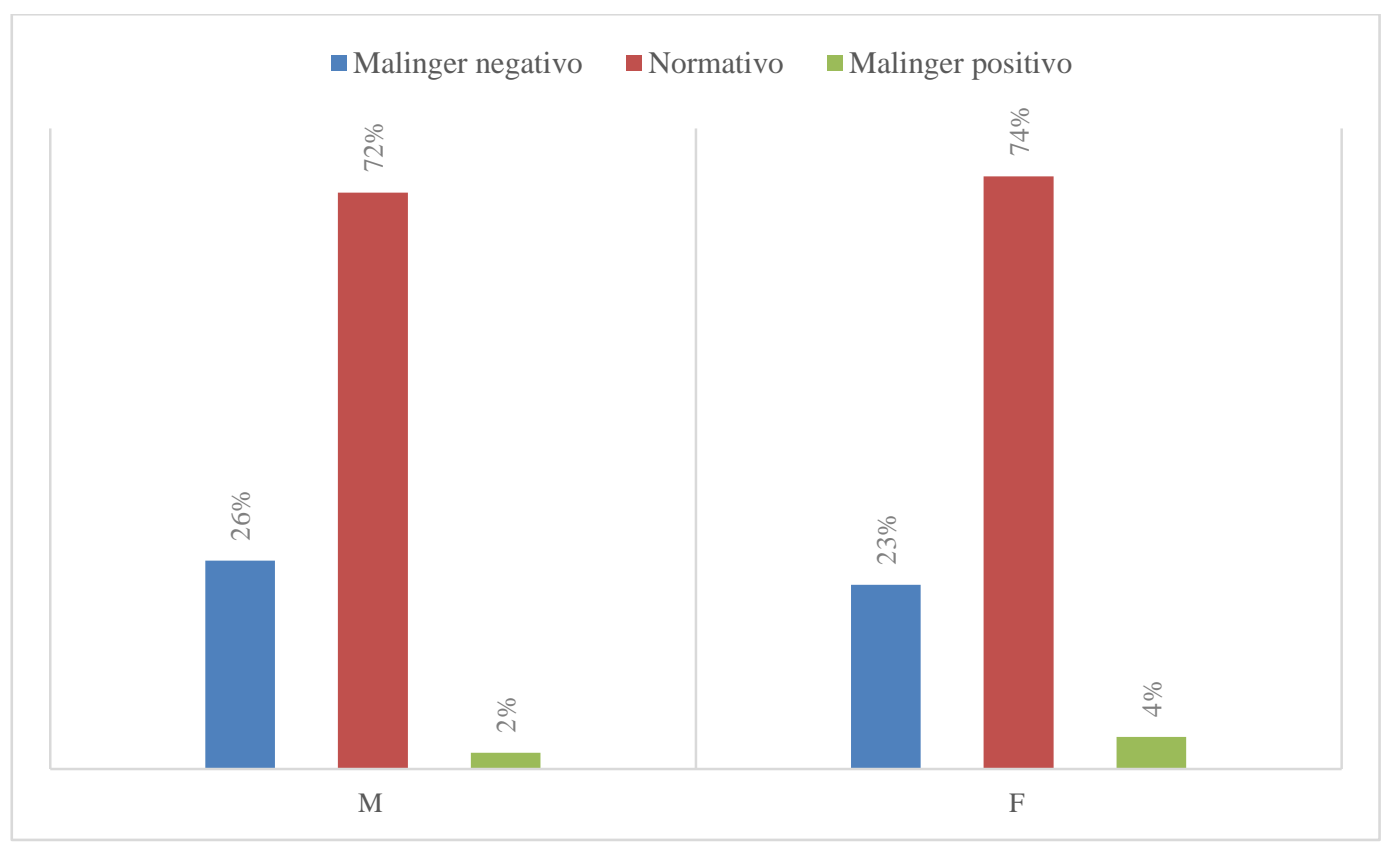

Figura 2. Atitude malinger e género.

Ao observar os resultados por categorias profissionais, notamos que os grupos com mais de $50 \%$ de participantes com uma atitude malinger negativa são os "especialistas de atividades intelectuais", "estudantes" e "reformados". Ainda com percentagens entre 30 e $40 \%$ de sujeitos que eventualmente dissimularam sintomatologia apresentam-se "representantes do poder legislativo", "militares", "trabalhadores da área da agricultura e pescas" e "operadores de instalações de 
máquinas". A atitude malinger positiva com maior destaque (20\%) verifica-se apenas na categoria "militares".

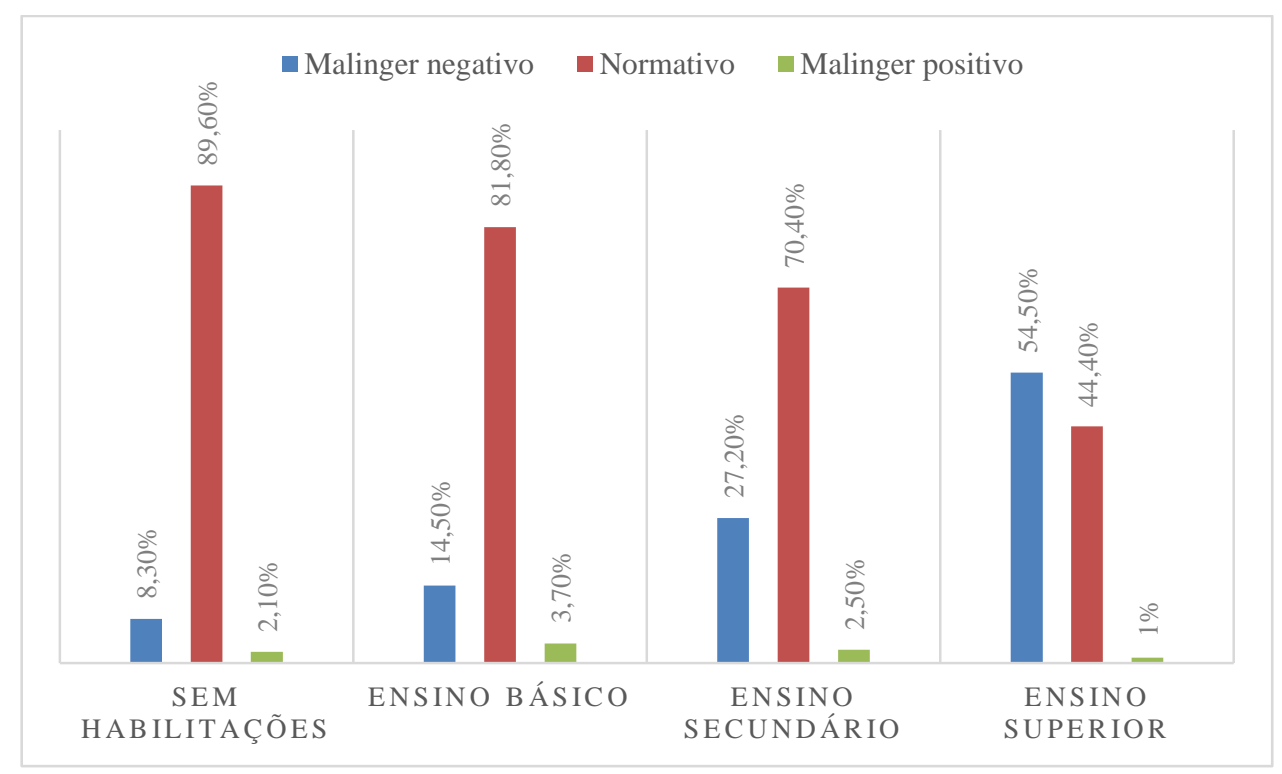

Figura 3. Atitude malinger e habilitações académicas.

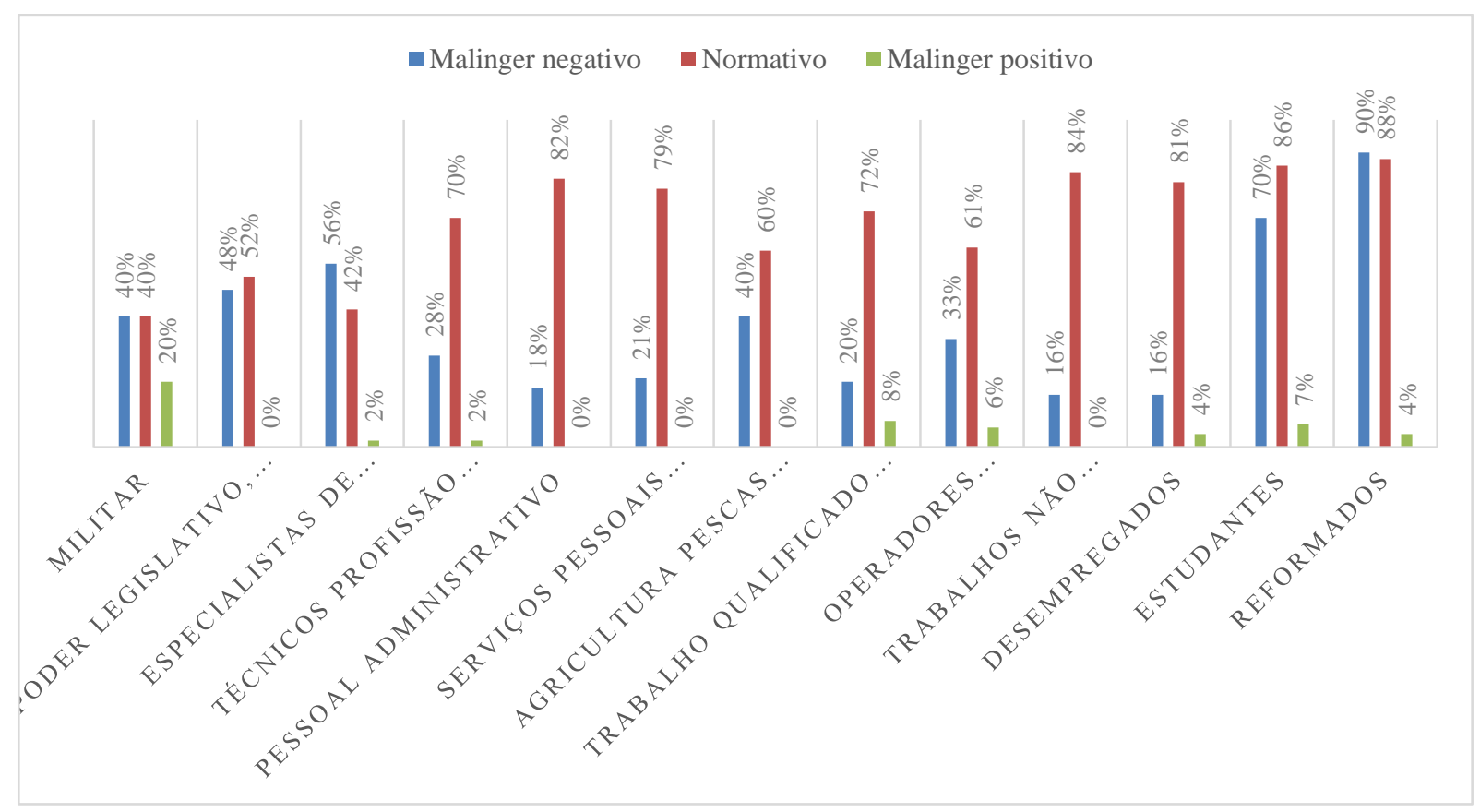

Figura 4. Atitude malinger e tipo de profissão.

O estado civil apresenta-se com resultados semelhantes entre os grupos. Apenas o grupo "Divorciado" teve um valor para a atitude malinger negativa acima dos $30 \%$. 


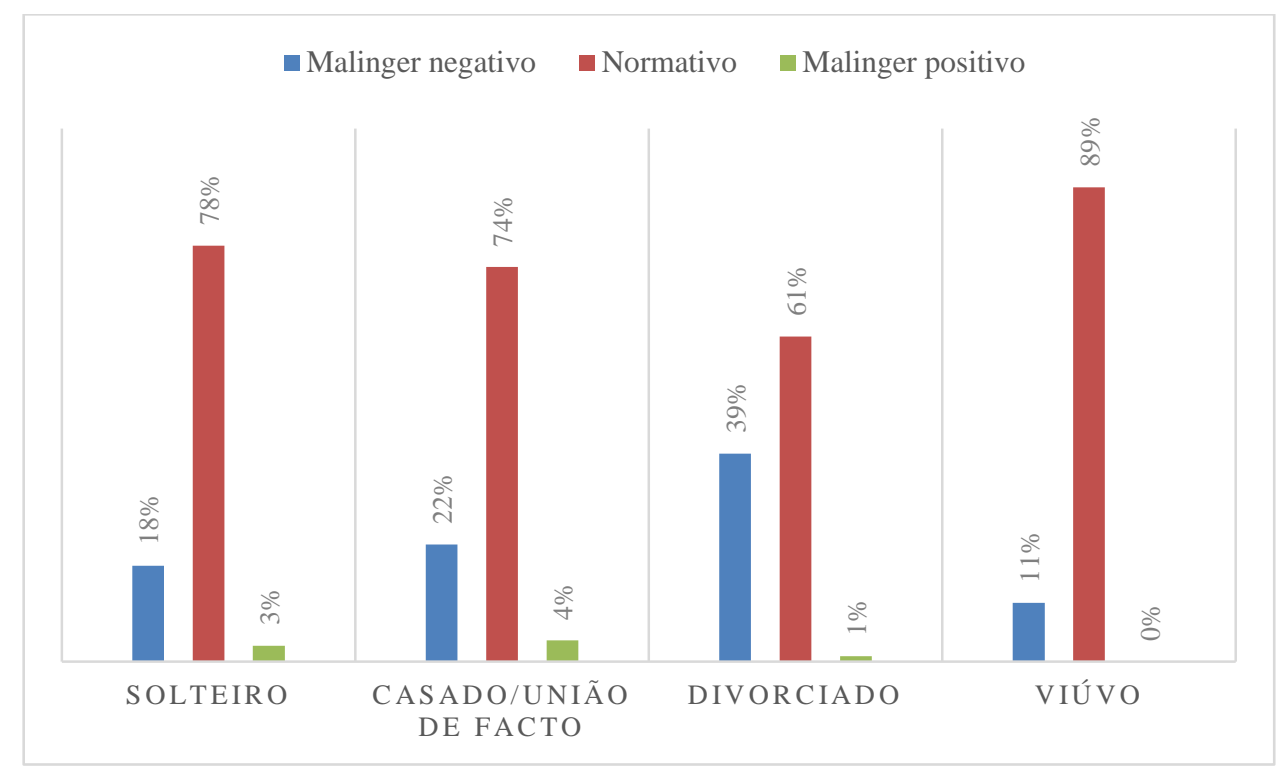

Figura 5. Atitude malinger e estado civil.

Ao observar a idade dos sujeitos e a forma como a atitude malinger se apresenta consoante essa variável, notamos que apenas no intervalo 47-61 existe um valor para a atitude malinger negativa acima dos $30 \%$.

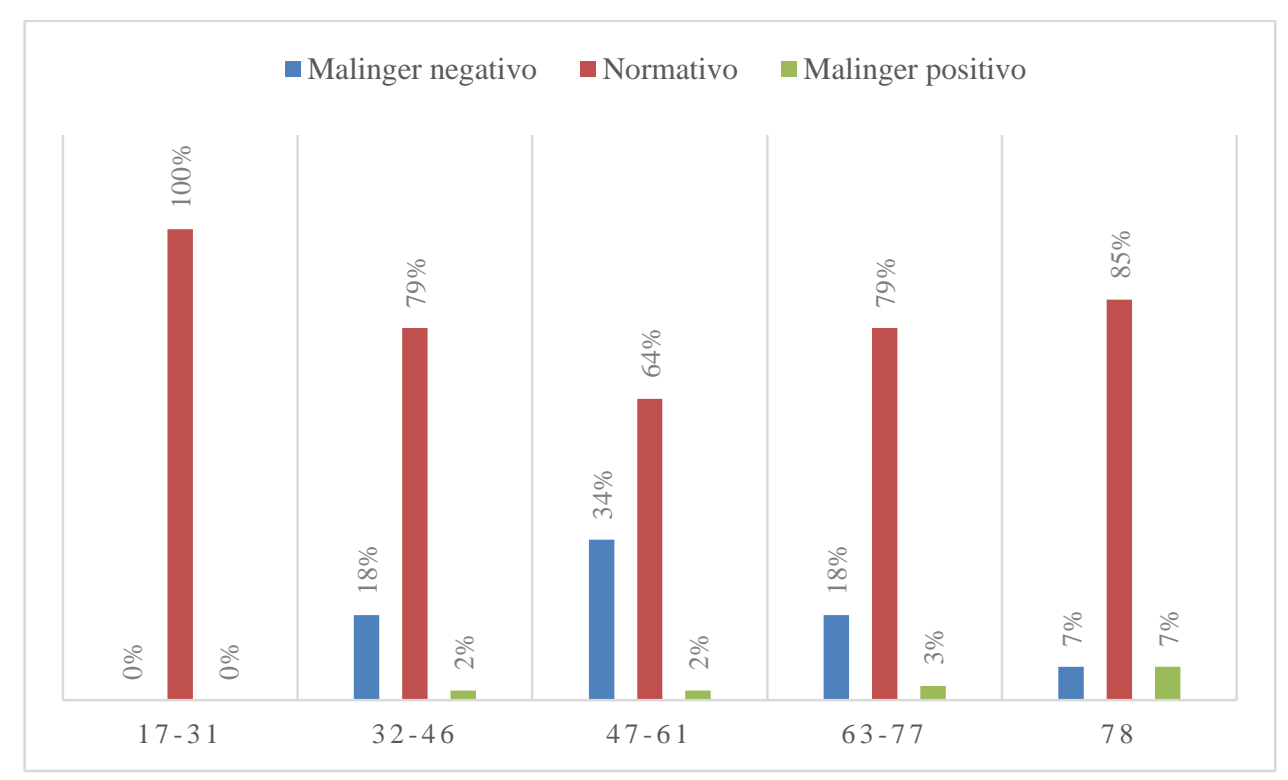

Figura 6. Atitude malinger e idade.

Relativamente à nacionalidade dos sujeitos avaliados, observa-se um maior valor para a atitude malinger negativa e positiva nos sujeitos não portugueses. 


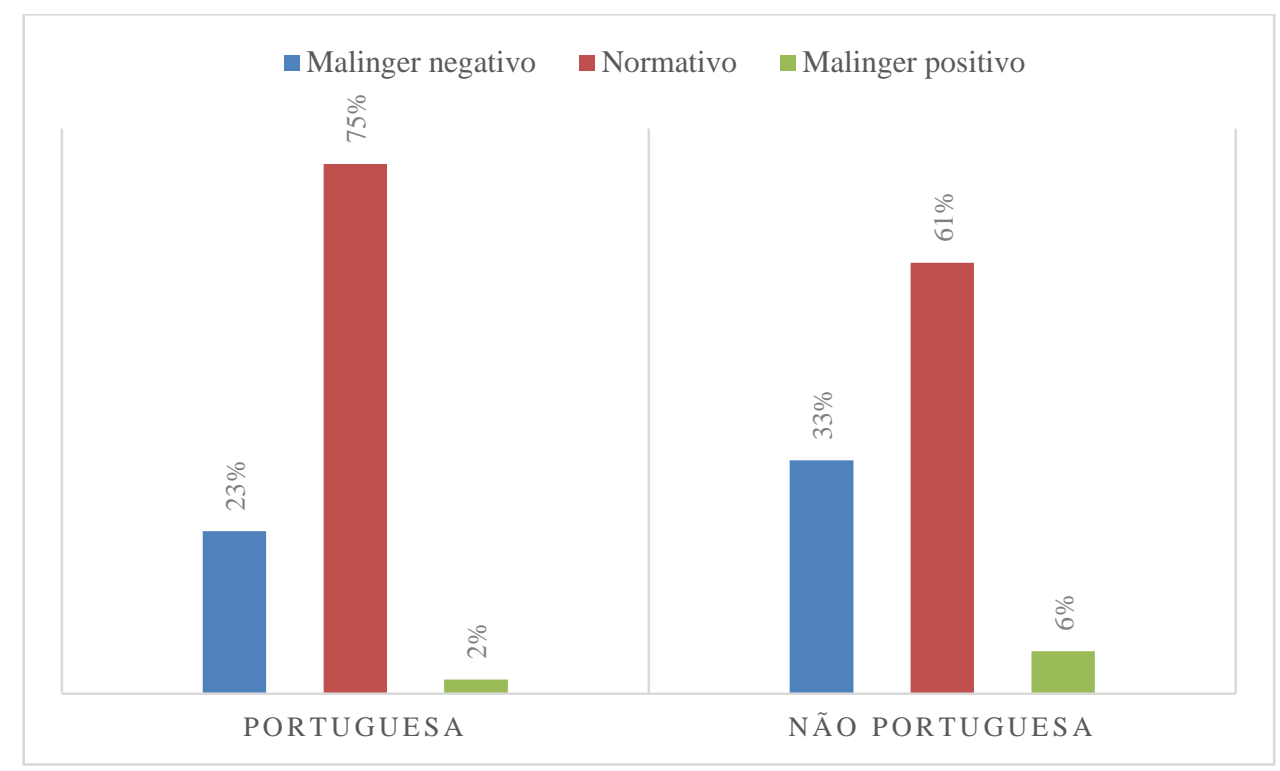

Figura 7. Atitude malinger e nacionalidade.

\subsection{Q1 (B)}

Relativamente às escalas clínicas com maior expressividade em sujeitos que apresentam uma atitude malinger, as três escalas clínicas mais elevadas em sujeitos com observada atitude malinger positiva $(\underline{f-k>11})$ são as escalas Sc [esquizofrenia], D [depressão] e Hy [histeria]. Relativamente às escalas com maior expressividade na atitude malinger negativa $(f-k \leq-15)$, observam-se as escalas clínicas Hy [histeria], D [depressão] e Pd [psicopatia]. Tais resultados podem ser vistos nas Figuras 8 e 9.

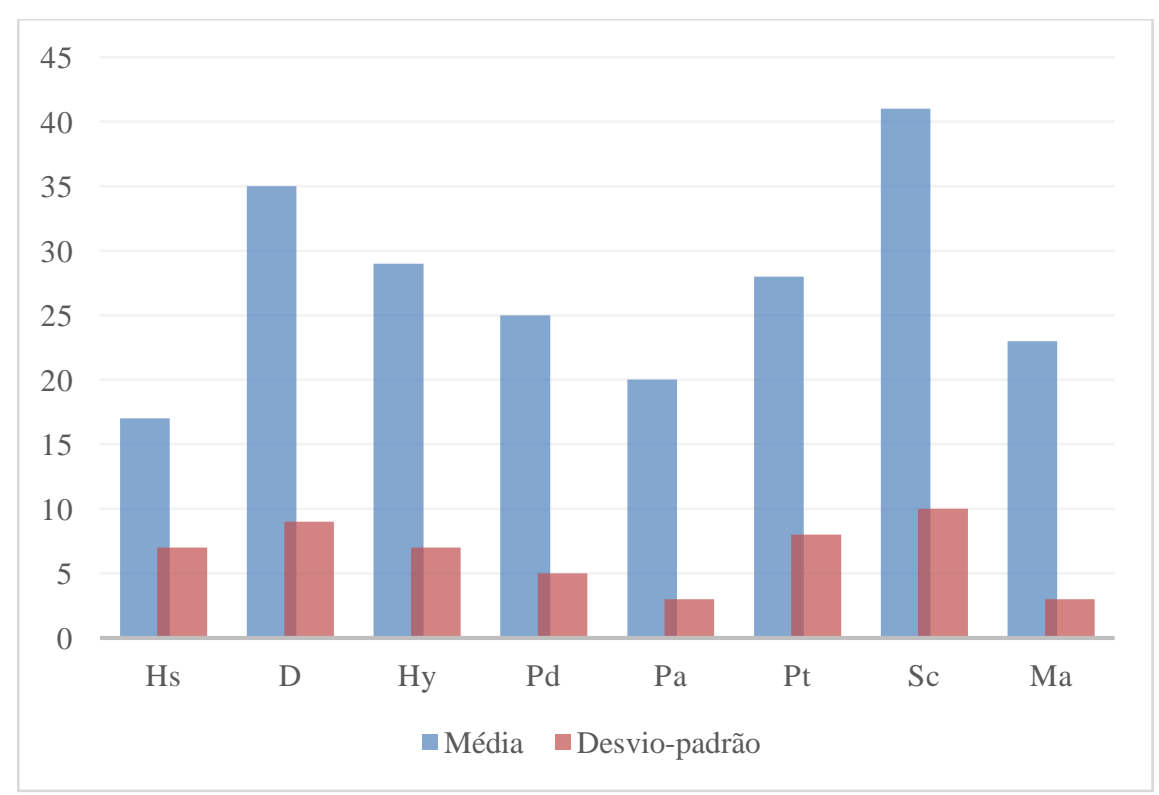

Figura 8. Escalas clínicas atitude malinger positiva $(n=13)$. 


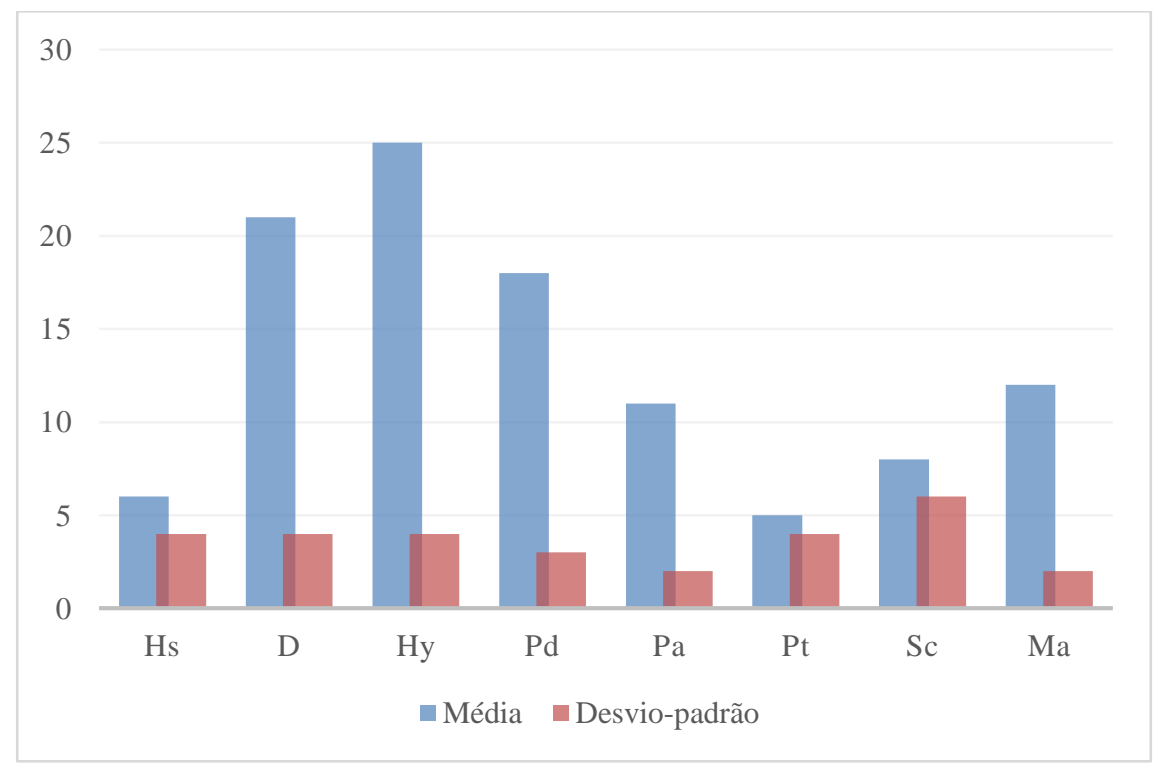

Figura 9. Escalas clínicas atitude malinger negativa $(n=115)$.

\subsection{Q2 (A)}

A análise da Tabela 1 permite observar diferenças significativas na presença de uma atitude malinger em perícias à personalidade quando comparadas com os outros tipos de perícia, com exceção das perícias para regulamentação do poder paternal.

Tabela 1. Comparação Índice $f-k$ nos diferentes tipos de perícia forense.

\begin{tabular}{|c|c|c|}
\hline Tipo de perícias & $\begin{array}{c}\text { P- } \\
\text { Values* }\end{array}$ & $\begin{array}{c}\text { Estatística } \\
\text { Z }\end{array}$ \\
\hline $\begin{array}{l}\text { perícia sobre a personalidade [art. 160 CPP] } \\
\text { vs. } \\
\text { perícia psicológica complementar à perícia psiquiátrica [art. } \\
\left.159^{\circ} \mathrm{CPP}\right]\end{array}$ & , 198 & $-1,288$ \\
\hline $\begin{array}{l}\text { perícia sobre a personalidade [art. 160 CPP] } \\
\text { vs. } \\
\text { perícia psicológica para avaliação do dano em sede de Direito } \\
\text { Penal e Civil }\end{array}$ & ,752 &,- 316 \\
\hline $\begin{array}{c}\text { perícia sobre a personalidade [art. 160 CPP] } \\
\text { vs. } \\
\text { perícia psicológica ao abrigo do artigo } 178^{\circ} \text { do Decreto-Lei } \\
\text { no314/78 (OTM) e da Lei no } 61 / 2008 \text { (Regime Jurídico do } \\
\text { Divórcio) }\end{array}$ & ,00 & $-3,566$ \\
\hline $\begin{array}{c}\text { perícia sobre a personalidade [art. 160 CPP] } \\
\text { vs. } \\
\text { perícia psicológica ao abrigo da Lei no } 147 / 99 \text { [Lei de Proteção }\end{array}$ & ,074 & $-1,789$ \\
\hline
\end{tabular}




\begin{tabular}{l|l|l}
\hline \multicolumn{1}{c|}{ de Crianças e Jovens em Perigo] } & & \\
\hline${ }^{\star} a=.05$ & & \\
$n=470$ & & \\
\hline
\end{tabular}

\subsection{Q2 (B)}

Relativamente a diferenças significativas entre a atitude malinger em processos de Regulamentação de Poder Paternal para com outros tipos de perícia, a análise da Tabela 2 apresenta os sujeitos neste tipo de processo como tendo uma atitude malinger destacada para com os outros tipos de processo, com exceção das perícias nos processos de Promoção e Proteção de Menores.

Tabela 2. Comparação Índice $f-k$ nos diferentes tipos de perícia forense.

\begin{tabular}{|c|c|c|}
\hline Tipo de perícias & $\begin{array}{c}\text { P- } \\
\text { Values* }\end{array}$ & $\begin{array}{c}\text { Estatística } \\
Z\end{array}$ \\
\hline $\begin{array}{c}\text { perícia psicológica ao abrigo do artigo } 178^{\circ} \text { do Decreto-Lei } \\
\text { no314/78 (OTM) e da Lei no } 61 / 2008 \text { (Regime Jurídico do } \\
\text { Divórcio) } \\
\text { vs } \\
\text { perícia psicológica complementar à perícia psiquiátrica [art. 159ำ } \\
\text { CPP] }\end{array}$ & .000 & -7.855 \\
\hline $\begin{array}{c}\text { perícia psicológica ao abrigo do artigo } 178^{\circ} \text { do Decreto-Lei } \\
\text { no314/78 (OTM) e da Lei no } 61 / 2008 \text { (Regime Jurídico do } \\
\text { Divórcio) } \\
\text { vs } \\
\text { perícia psicológica para avaliação do dano em sede de Direito } \\
\text { Penal e Civil }\end{array}$ & . 000 & -3.883 \\
\hline $\begin{array}{c}\text { perícia psicológica ao abrigo do artigo } 178^{\circ} \text { do Decreto-Lei } \\
\text { ํo314/78 (OTM) e da Lei no } 61 / 2008 \text { (Regime Jurídico do } \\
\text { Divórcio) } \\
\text { vs } \\
\text { perícia sobre a personalidade [art. } 160^{\circ} \text { CPP] }\end{array}$ & .000 & -3.566 \\
\hline $\begin{array}{l}\text { perícia psicológica ao abrigo do artigo } 178^{\circ} \text { do Decreto-Lei } \\
\text { no314/78 (OTM) e da Lei no } 61 / 2008 \text { (Regime Jurídico do } \\
\text { Divórcio) } \\
\text { vs } \\
\text { perícia psicológica ao abrigo da Lei no } 147 / 99 \text { [Lei de Proteção } \\
\text { de Crianças e Jovens em Perigo] }\end{array}$ & .054 & -1.930 \\
\hline
\end{tabular}




\section{$4.5 Q 3$}

A análise da regressão linear simples ${ }^{25}$ apresenta o valor de $13.6 \%$ como sendo a variabilidade da variável índice $f=k$ explicada de forma significativa pelo índice de Q.I..

Tabela 3. Correlação Índice f-k vs Índice Q.I.

\begin{tabular}{c|c|c|c|c|c}
\hline \hline Modelo & $\mathrm{R}$ & $\mathrm{R}$ & $\mathrm{R}$ (Ajustado) & $\mathrm{SD}$ & $\begin{array}{c}\text { Durbin- } \\
\text { Watson }\end{array}$ \\
\hline 1 & .372 & .139 & .136 & 8.464 & 2.073 \\
\hline$n=346$ & \multicolumn{5}{l}{} \\
\hline \hline Anova da regressão linear para rejeição de $\mathrm{H} 0$ & $\mathrm{~F}$ & \\
\hline Modelo & $\begin{array}{c}\text { Soma dos } \\
\text { Quadrados }\end{array}$ & $\mathrm{df}$ & $\begin{array}{c}\text { Média } \\
\text { Quadrado }\end{array}$ & & \\
\hline Regressão & 3908.922 & 1 & 3908.922 & 54.555 & $.000^{*}$ \\
\hline Residual & 24289.869 & 339 & 71.652 & & \\
\hline Total & 28198.792 & 340 & & & \\
\hline${ }^{*} a=.05$ \\
$n=346$
\end{tabular}

\section{Discussão}

Uma vez findada a análise de resultados, será importante discutir a mesma. Neste sentido, os resultados observados nas questões de investigação têm dois tipos de características que importam referir. Primeiramente, uma considerável parte do trabalho estatístico é apenas descritiva, carecendo de elegância científica que seja digna de um trabalho de investigação superior. Embora tendo a capacidade para tecer esta consideração, consideramos que é deveras importante também observar o interesse que estes dados - pouco elegantes - se possam fazer acompanhar. São poucos os estudos que em Portugal têm sido realizados na área da avaliação psicológica em geral, e em particular na área da avaliação da atitude malinger. É ainda mais diminuto o número de estudos que tendo o foco anterior referido, tem como população de estudo uma amostra totalmente forense. Drob, Meehan e Waxman ${ }^{21}$ referem a necessidade de se realizarem estudos que tenham como amostra populações forenses a fim de não se promover eventuais enviesamentos cognitivos, criando falsas expectativas nos profissionais que avaliam sujeitos a nível forense, bem como profissionais da área do direito ou outras adjacentes a uma avaliação pericial. Sobre este ponto, achamos que as instituições académicas conjuntamente com as instituições públicas, nomeadamente as de carácter de 
avaliação legal, deveriam capacitar-se para o investimento ao nível da investigação e de promoção de um conhecimento novo e refrescado. Não só no trabalho conjunto entre academia e serviço público, ou até mesmo privado, mas também na criação de comités de ética que possam promover a autonomia científica de profissionais.

Relativamente à caracterização sociodemográfica e sua associação à atitude malinger, temos a capacidade para encontrar um espaço de humildade e assumir a limitada elegância estatística de se ter realizado apenas uma análise descritiva. Não havendo a possibilidade de outro tipo de análises, a premissa foi única e exclusivamente a de descrever a população. Conhecendo a população a quem se realizaram avaliações periciais no passado poderemos ter uma baseline para estudos futuros. Não se encontraram resultados com uma relevância acima do normal [i.e. resultados com diferenças de $>50 \%$ entre sub-grupos]. No entanto, é interessante observar-se que alguns sub-grupos parecem ser mais predispostos a atitudes malinger, como o caso de sujeitos com habilitações académicas superiores. Este ponto vai ao encontro da profissão com a maior atitude malinger negativa ser a categoria de "especialistas de atividades intelectuais científicas". Acreditamos que não será possível, nem tão pouco benéfico, criar um perfil do sujeito malinger. Embora com os presentes resultados fosse possível elencar algumas características, esse trabalho além de carecer de algum suporte científico, iria também ser, eventualmente, fonte de enviesamentos cognitivos, algo que a investigação apresenta como uma das maiores limitações dos profissionais da especialidade. Edens, Poythress e Watkins-Clay ${ }^{26}$ referiram no seu trabalho que o uso de instrumentos específicos para avaliação da atitude malinger [eg. SIMS e SIRS] quando comparados com instrumentos de avaliação de personalidade [eg. $\mathrm{PAl}$ ] tiveram uma performance mais limitada na distinção entre pacientes psiquiátricos e pacientes suspeitos de apresentar uma atitude malinger. Os nossos resultados parecem inclinar-se para conclusões semelhantes a estas. $\mathrm{Na}$ nossa amostra, é importante apresentar que a atitude malinger está, de facto, presente na avaliação forense. Observamos que quase $30 \%$ da nossa amostra apresentou algum tipo de atitude malinger, indo ao encontro de outros estudos onde profissionais foram avaliados sobre a preponderância de casos onde se observava presença de atitudes malinger na amostra avaliada ${ }^{1,27,28}$. Esta realidade motiva-nos a solicitar aos poderes de decisão que promovam ações de investigação científica na área. Ainda sobre o resultado anterior, a atitude malinger negativa é a que se 
observa com maior preponderância. Isto poderá sugerir que a forma como foi feita a análise da atitude malinger é limitada por a prova usada não ser específica para este tipo de avaliação, mas para a avaliação de traços de personalidade, ou eventualmente sugerir que a prova é fina a distinguir a atitude malinger positiva da atitude malinger negativa conforme os resultados de Edens, Poythress e WatkinsClay (26).

Os traços de personalidade que se observam nos dois tipos de atitude malinger poderão sugerir quais os traços que mais poderão ser tentados de falsear/fingir, no entanto já se observou que poderão também sugerir pedidos de ajuda mediante situações de desespero conforme outros estudos demonstraram ${ }^{4,29}$, i.e., momentos onde a dor psicológica é tão intensa que embora a atitude de fingir o comportamento esteja presente, algo mais haverá a ter em consideração. Sobre este ponto, sugere-se que ao nível institucional sejam feitas avaliações com tempo suficiente, condições adequadas e onde os tribunais tenham em consideração que o trabalho da psicologia forense se prende com a aplicação de um conjunto de tarefas com carácter científico e metodológico.

Relativamente aos resultados observados nas questões 2, acreditamos que a necessidade de se obter mais informação sobre a atitude em cada tipo de processo jurídico é premente. Se conseguirmos considerar que cada sujeito terá a sua forma de pensar e agir mediante cada tipo de contexto, então será pertinente sugerir que a atitude de falsear o comportamento terá algum tipo de correlação com o tipo de processo jurídico em que o sujeito esteja afeto. Os nossos resultados vão ao encontro dessa suposição com diferenças significativas entre os processos jurídicos e os valores dos índices $f-k$ observados nas avaliações dos mesmos. Importa também referir que a avaliação da atitude malingering é uma tarefa que é parte da avaliação psicológica, i.e., não partilhamos da opinião de que em determinado tipo de processo/perícia se tente procurar traços e/ou atitudes falsas, mas sim que se tenha presente a necessidade de observar essa possibilidade durante a avaliação a fim de evitar enviesamentos cognitivos como por exemplos enviesamentos de confirmação e/ou ancoragem.

Finalmente a terceira questão de investigação propõe-nos a ter alguma precaução na sua observação absoluta. Tendo os resultados apontado para uma correlação significativa entre o índice de Q.I. e a atitude malinger, não gostaríamos de passar a ideia de que a inteligência é algo que per se explicará a atitude de 
falsear um comportamento, mas sim uma competência que estará associada a determinados tipos de malingering. Uma observação que nos levanta curiosidade para um eventual estudo futuro será a associação entre a inteligência, os graus académicos e o tipo de atitude malinger [positiva ou negativa]. No entanto, no presente trabalho apenas temos a capacidade para enunciar com rigor científico que a inteligência parece ter um papel importante na capacidade de tentar falsear um comportamento.

O uso de um diagnóstico de atitude malinger observou no passado algumas críticas relativamente à forma como estariam incluídos alguns dos seus critérios em versões anteriores do $\mathrm{DSM}^{30}$, promovendo algumas alterações em versões recentes do mesmo manual ${ }^{31}$. As maiores diferenças prendem-se com a forma como a atitude malinger poderá ser interpretada e confundida com a atitude de feigning [i.e. fingir] sintomatologia. Do interesse desta distinção, acreditamos que a maior para a comunidade profissional será a de conseguir diferenciar a atitude malinger por exemplo de perturbações factícias [i.e. fingimento consciente com um objetivo interno - assumir uma doença] ou a perturbação de conversão [i.e. sintomas médico incompatíveis criados inconscientemente]. Para este tipo de situações, importará referir que a nível prático, poderão os profissionais de psicologia forense e os seus relatórios, e os profissionais de direito que irão suportar-se com tais documentos para o seu trabalho, enfrentarem discussões relativas à presença ou não de situações de inimputabilidade. Em Portugal, é o número 1 do artigo 20ํ do Código Penal $^{32}$ que refere a inimputabilidade de uma pessoa pela sua incapacidade de avaliar ou se autodeterminar perante a ilicitude da prática do seu ato por motivo de anomalia psíquica. Estando os profissionais da psicologia forense confrontados com sujeitos que poderão falsear [quer produzindo ou ocultando] quadros sintomatológicos de carácter inconsciente, levanta-se a questão relativa à sua capacidade de assumir o designado dolo jurídico. Sobre esta necessidade de reflexão, acreditamos que não se deverá apenas cingir o trabalho do psicólogo forense a criar um diagnóstico, do tipo binomial sim ou não, mas sim conceptualizar, descrever e compreender as circunstâncias externas e internas, inerentes ao indivíduo, em que o acontecimento ocorreu. Em outras palavras, caberá ao tribunal fundamentar-se e tomar decisões de carácter binomial, enquanto que à psicologia forense, apenas se deverá esperar a compreensão e caracterização cognitiva e comportamental de sujeitos avaliados. 
Profissionais de psicologia forense deverão ter em consideração potenciais enviesamentos cognitivos em momentos de avaliação com os obstáculos que a análise da atitude falsa, nomeadamente da atitude malinger contém ${ }^{21}$. LeBourgeois ${ }^{33}$ apresentou um quadro bastante sintético para se realizar uma avaliação da atitude malinger e a sua distinção de perturbações factícias, somatoformes bem como sintomatologia médica/psiquiátrica válida. Para que profissionais de psicologia forense consigam fazer-se valer de uma metodologia como a elaborada por LeBourgeois $^{33}$, necessitam as instituições de promover condições para tal, bem como criar espaço para eventualmente discutir as diferenças e benefícios entre 0 trabalho exclusivo do profissional de psicologia forense a nível público ou porventura também a nível privado. Outra necessidade que observamos é a de capacitar os profissionais com instrumentos válidos, mas mais importante fidedignos e precisos. Existem testes psicométricos que foram construídos para a análise da atitude malinger ou de comportamento de simulação (eg. SIMS, SIRS) mas que têm vindo a apresentar limitações na sua capacidade de distinção quando comparados com o uso de sub-escalas de instrumentos de avaliação de personalidade ${ }^{26}$. Finalmente a necessidade de se ter a avaliação psicológica como uma parte de um trabalho multidisciplinar a nível organizacional. Não será possível ter o profissional de psicologia forense limitado ao espaço da psicologia apenas. Linhas orientadoras de trabalho multidisciplinar deverão ser implementadas e coordenadas por quadros de direção. O saber da psicologia agregado ao saber da medicina legal, do trabalho social e do direito acrescentará mais valor quanto maior for a partilha entre profissionais de áreas diferentes assim como o uso de fontes colaterais sempre que se entenda ser necessário. A atividade de profissionais de psicologia, em contexto pericial, contempla um conjunto de metodologias sustentadas por modelos teóricos que culminam na produção de um relatório pericial complementar à fundamentação da magistratura. Através de meios de avaliação psicológica, este relatório pericial, tem como objetivo não apenas, e exclusivamente, a compreensão de um sujeito, como a área clínica almeja, mas sim a avaliação e informação aos profissionais da justiça sobre variáveis relativas à pessoa em exame tais como traços de personalidade, fatores de risco e fatores de proteção, vulnerabilidades mentais e cognitivas, psicopatologias, entre outras. 


\section{Limitações e estudos futuros}

Assim como acreditamos que os resultados por nós apresentados têm a capacidade de aumentar o conhecimento sobre a área da atitude malinger, também a humildade nos promove o olhar para os mesmos resultados e apontar algumas limitações. Ao nível das ferramentas analisadas, nomeadamente o uso do Mini-Mult, poderemos referir que esta é uma ferramenta em que o seu investimento científico é q.b. limitado. No entanto a justificativa para o seu uso prende-se com ter sido o instrumento psicométrico com maior utilização nas instituições onde se realizou a recolha de dados. Este ponto permite-nos referir novamente a necessidade de novos estudos de validação de ferramentas específicas para a avaliação da atitude malinger, e acrescentar que esses mesmos estudos sejam realizados com populações totalmente forenses, como o caso do nosso trabalho. É importante referir que os dados recolhidos foram exclusivamente dados de perícias forenses, não nos foi possível observar dados/documentos de outras áreas. Isto leva-nos a crer que o trabalho multidisciplinar ainda não é algo implementado, e por isso limitativo ao nível de estudos como o nosso. A ideia de que a nossa amostra não é totalmente de sujeitos de nacionalidade portuguesa proporciona a sugestão da necessidade de estudos também com o foco em emigrantes em situação de avaliação forense. A cultura é algo extremamente importante e o seu isolamento quer por ignorância ou por falta de tempo poderá reduzir bastante a capacidade de compreender o sujeito em avaliação ou até mesmo impossibilitar em parte ou totalmente a avaliação por motivos linguísticos. Finalmente a possibilidade de ter entidades disponíveis para colaborar com investigadores. No presente caso tal não aconteceu, mas ainda assim o trabalho burocrático fez-se notar. A possível sugestão é a criação de pontos de contacto dentro de cada instituição para que se possa realizar mais investigação com os dados que são diariamente recolhidos.

\section{Referências}

1. Singh J, Awasthi A, Grover S. Malingering of Psychiatric Disorders: A Review. German Journal of Psychiatry. 2007;10(4):126-32.

2. Kluck M-L, Westhoff K. Applied fields: Forensic. In: Fernández-Ballesteros R, editor. Encyclopedia of Psychological Assessment. London: Sage Publications; 2003. p. 59-62 
3. Gonçalves FV. Avaliação Psicológica em Contexto Forense: Desafios e Diálogos na Determinação da Mentira. In: Paulino M, Alho L, coordenadores. Comportamento Criminal e Avaliação Forense. Pactor: Lisboa; 2018. p. 21-38.

4. Gonçalves, FV. Dissimulaçao do desespero: Uma Realidade da Regulação das Responsabilidades Parentais em Portugal. Direito em Debate. 2015;24(43):47-61.

5. Agulhas R, Anciães A. Casos Práticos em Psicologia Forense. Lisboa: Edições Silabo; 2014.

6. Rogers R. An introduction to response styles. Clinical assessment of malingering and deception. New York, NY: Guildford Press; 2008. pp. 3-13.

7. Rogers R, Correa AA. Determinations of malingering: Evolution from case-based methods to detection strategies. Psychiatry, Psychology and Law. 2008. 15, 213-23. https://doi.org/10.1080/13218710802014501

8. American Psychiatric Association. Diagnostic and statistical manual of mental disorders $5^{\text {th }}$ ed. Washington, DC; 2013. https://doi.org/10.1176/appi.books.9780890425596

9. Rogers R, Salekin RT, Sewell KW, Goldstein A, Leonard K. A comparison of forensic and nonforensic malingerers: a prototypical analysis of explanatory models. Law and human behavior, 1998;22:353-67. https://doi.org/10.1023/A:1025714808591

10. Wygant DB, Anderson JL, Sellbom M, Rapier JL, Allgeier LM et al. Association of the MMPI-2 restructured form (MMPI-2-RF) validity scales with structured malingering criteria. Psychological Injury and Law. 2011;4(1):13-23. https://doi.org/10.1007/s12207$\underline{011-9098-z}$

11. Resnick PJ, West S, Payne JW. Malingering on posttraumatic disorders. In: Rogers R, editor. Clinical assessment of malingering and deception. New York, NY: Guildford Press; 2008. p. 109-27.

12. Taylor S, Fruch BC, Asmundson GJG. Detection and management of malingering in people presenting for treatment of posttraumatic stress disorder: Methods, obstacles and recommendations. Journal of Anxiety Disorders. 2006;21(1):22-41. https://doi.org/10.1016/j.janxdis.2006.03.016

13. Kitchen R. Investigating benefit fraud and illness deception in the United Kingdom. In: Halligan PW, Bass C, Oakley DA, editors. Malingering and illness deception. New York, NY: Oxford University Press; 2003. p. 313-322

14. Gonçalves FV, Pires A, Vila-Real A. O Sujeito Simulador na Avaliação Psicológica Forense. Direito em Debate. 2017;26(47):332-49. https://doi.org/10.21527/2176$\underline{6622.2017 .47 .332-349}$

15. Tarescavage AM, Wygant DB, Gervais RO, Ben-Porath YS. Association between the MMPI-2 Restructured Form (MMPI-2-RF) and malingered neurocognitive dysfunction 
among non-head injury disability claimants. The Clinical Neuropsychologist. 2013;27(2): 313-35. https://doi.org/10.1080/13854046.2012.744099

16. Sellbom M, Toomey JA, Wygant DB, Kucharski LT, Duncan S. Utility of the MMPI-2-RF (Restructured Form) validity scales in detecting malingering in a criminal forensic setting: A known-groups design. Psychological Assessment. 2010;22(1):22-31. https://doi.org/10.1037/a0018222

17. Rogers R, Vitacco MJ, Kurus SJ. Assessment of malingering with repeat forensic evaluations: Patient variability and possible misclassification on the SIRS and other feigning measures. Journal of the American Academy of Psychiatry and the Law. 2009;38(1):109-14.

18. Hathaway SR, McKinley JC. A multiphasic personality schedule (Minnesota): I. Construction of the schedule. The Journal of Psychology. 1940;10(2):249-54. https://doi.org/10.1080/00223980.1940.9917000

19. Kincannon JC. Prediction of the standard MMPI scale scores from 71 items: the MiniMult. Journal of consulting and clinical psychology. 1968;32(3):319-25. https://doi.org/10.1037/h0025891

20. Tracy DK, Rix KJ. Malingering mental disorders: Clinical assessment. BJPsych Advances. 2017;23:27-35. https://doi.org/10.1192/apt.bp.116.015958

21. Drob SL, Meehan KB, Waxman SE. Clinical and conceptual problems in the attribution of malingering in forensic evaluations. The journal of the American Academy of Psychiatry and the Law. 2009;37(1):98-106.

22. Raven J, Raven JC, Court JH. Raven Manual: Section 4, Advanced Progressive Matrices, 1998 Edition. Oxford, UK: Oxford Psychologists Press Ltd; 1998.

23. Cunha JA. MMPI: Escalas de validade e escalas clínicas. In: Cunha JÁ, editor, Psicodiagnóstico-V (pp. 454-479). (5ª edição revisada e ampliada). Porto Alegre: Artes Médicas; 2008. p. 454-79.

24. IBM Corp. Released. IBM SPSS Statistics for Windows, Version 25.0. Armonk, NY: IBM Corp; 2017

25. Marôco JP. Análise estatística com o PASW Statistics (ex-SPSS). Ed. 1. Report Number: Pêro Pinheiro; 2010

26. Edens JF, Poythress NG, Watkins-clay MM. Detection of Malingering in Psychiatric Unit and General Population Prison Inmates: A Comparison of the PAI, SIMS, and SIRS. Journal of Personality Assessment. 2007;88(1):3342. https://doi.org/10.1080/00223890709336832

27. Mason AM, Cardell R, Armstrong M. Malingering Psychosis: Guidelines for Assessment and Management. Perspectives in Psychiatric Care. 2014;50(1):51-7. https://doi.org/10.1111/ppc. 12025 
28. Mittenberg W, Patton C, Canyock EM, Condit DC. Base rate of malingering and symptom exaggeration. Journal of Clinical and Experimental Neuropsychology. 2002;24(8):1094-102. https://doi.org/10.1076/jcen.24.8.1094.8379

29. Hall RCW, Hall RCW. Malingering of PTSD: forensic and diagnostic considerations, characteristics of malingerers and clinical presentations. General Hospital Psychiatry. 2006;28(6):525-35. https://doi.org/10.1016/j.genhosppsych.2006.08.011

30. Berry DTR, Nelson NW. DSM-5 and malingering: A modest proposal. Psychological Injury and Law. 2010;3(4):295-303. https://doi.org/10.1007/s12207-010-9087-7

31. Wallace ER, Garcia-Willingham NE, Walls BD, Bosch CM, Balthrop KC, Berry DTR. A meta-analysis of malingering detection measures for attention-deficit/hyperactivity disorder. Psychological 2019;31(2):265-70. https://doi.org/10.1037/pas0000659

32. Código Penal Português. Coimbra: Almedina; 2007.

33. Lebourgeois HW. Malingering: Key points in assessment. Psychiatric Times. 2007;24(4):21-9. 